\title{
Temporal dynamics of endogenous attentional modulation without cue awareness
}

\author{
Yuma Osako ${ }^{1,4, *}$, Shota Murai ${ }^{2,4}$, Jun Shimpaku ${ }^{3,4}$ and Kohta I. Kobayasi2,"* \\ ${ }^{1}$ Laboratory of Neural Information, Graduate School of Brain Science, Doshisha \\ University, Kyotanabe, Kyoto 610-0394, Japan \\ ${ }^{2}$ Graduate School of Life and Medical Sciences, Doshisha University, Kyotanabe, \\ Kyoto, 610-0321, Japan. \\ ${ }^{3}$ Faculty of Life and Medical Sciences, Doshisha University, Kyotanabe, Kyoto, 610- \\ 0321, Japan. \\ ${ }^{4}$ Co-first authors \\ *yyosako@gmail.com (Y.O.), kkobayas@mail.doshisha.ac.jp (K.K.)
}

\begin{abstract}
Invisible visual stimuli can regulate our broad cognitive performance in the pursuit of current goals. Endogenous spatial attention is an important modulator of cognitive performance, and it can be triggered by unconscious cues. However, how its effect changes with time remains unclear. Here, we show that endogenous attention was triggered by an arrow-cue whose direction participants reported being unaware of but which affected the task performance in a time-dependent manner. Participants were asked to remember the directions of eight Landolt c rings (target memory array) after arrow-cue presentation, which was designed to orient their attention to a certain $\mathrm{c}$ ring. Then, we applied a delay, ranging from $83 \mathrm{~ms}$ to $1000 \mathrm{~ms}$, between the arrow-cue and the target memory array presentation (the possible delays were equally spaced on a logarithmic scale). The attentional effect was greater for the 83,183, 250 and 333 ms delays than the other six possible delays. In contrast, its effect was maintained irrespective of the delay when the participants reported being aware of the cue direction. Thus, awareness of arrow-cue direction was necessary to maintain endogenous attentional modulation, and its modulation without arrow-cue direction awareness was limited in a time-dependent manner.
\end{abstract}

\section{Introduction}

A visual stimulus falling outside our awareness can bias our conscious experience ${ }^{1,2}$ and behavioural responses ${ }^{3,4}$. These phenomena have been reported in blindsight patients who suffer from cortical blindness due to lesions in the primary visual cortex (V1). They can 
discriminate or respond to visual stimuli even though they cannot consciously see them ${ }^{5-}$ 7. This unconscious processing of invisible stimuli was also reported in healthy human participants in cases of masking ${ }^{8}$, binocular rivalry ${ }^{9}$, inattentional blindness ${ }^{10}$, motioninduced blindness ${ }^{11}$ and continuous flash suppression ${ }^{12}$. Such unconscious visual information can affect performance in a broad range of cognitive tasks, such as object recognition ${ }^{13,14}$ and decision-making ${ }^{15-18}$. This unconscious processing may accelerate our cognitive and informative life to improve and promote the efficiency of conscious performance.

Spatial attention is one of the main factors that can improve or promote the processing of visual stimuli ${ }^{19}$. Spatial attention is triggered by visual stimuli and is suggested to modulate the neural response at the early and late stages of stimulus processing ${ }^{20-23}$. Unconscious stimuli also trigger the shift of spatial attention ${ }^{24-28}$. However, most studies demonstrated that these effects were elicited by peripheral or spatially compatible cues, which could drive the shift of spatial attention in an exogenous manner. A masked prime presented in a central position can nonetheless affect the shift of spatial attention to the peripheral space $e^{29,30}$. These findings indicate that an unconscious prime can be used to handle the shift of spatial attention in an endogenous manner. Clarifying the neural correlates of endogenous spatial attention, which unconscious visual stimuli trigger, is important in understanding the neural mechanisms of unconscious performance. However, little is known about whether the effect of such spatial attention is temporally changed, as well as when the unconscious shift of attention occurs after subliminal visual stimulus presentation. To understand the temporal dynamics of these unconscious modulations in performance, it is necessary to address the temporal dissociation of endogenous attentional effects with and without cue awareness.

Thus, we utilized the effect of spatial attention on encoding visual stimuli into visual short-term memory (VSTM). For example, when participants attend to a specific location in their visual field, the target stimulus at that location is more likely to be encoded into VSTM than other target stimuli at unattended locations ${ }^{31-33}$. Then, to test the temporal dynamics of attentional effects, we applied a pre-cueing paradigm ${ }^{19}$, in which an attentional arrow-cue was presented before a delay of 83-1000 ms until a target memory array presentation (eight Landolt c rings shaping an imaginary circle). The subjects were instructed to encode the visual memory array into VSTM, and following the retention interval $(100 \mathrm{~ms})$, a probe cue was presented at the target memory array. The participants answered in which direction the Landolt $\mathrm{c}$ ring at that position pointed. The attentional arrow-cue was intended to modify the location of attentional space, and we rendered its arrow direction invisible by using forward and backward masking. 


\section{Methods}

\section{Participants and Apparatus.}

A total of twenty-two healthy adults (13 males, 9 females, aged 21-27) participated in this study voluntarily. All participants gave informed consent and were paid for their participation. All experiments were performed in accordance with the guidelines for experiments at Doshisha University with the approval of the Research Committee of Doshisha University. All the data were anonymously treated, and the private information was prevented from leakage.

The experiment was programmed in MATLAB using Psychotoolbox ${ }^{34}$ and was conducted in a sound-proof and darkened room with only the monitor producing light. All visual stimuli were generated by a $60 \mathrm{~Hz}$-refresh-rate monitor and displayed at a resolution of $1920 \times 1080$ (FG2421, EIZO, Ishikawa). The masking stimuli were synthesized with random RGB dots. The masking procedure was one of the common ways to render cue blindness ${ }^{35,36}$. The monitor used a grey background to reduce afterimages. All responses were collected by inputs on a standard keyboard. All participants set their jaw on a chin rest to stabilize their eye position and to keep a viewing distance of approximately $60 \mathrm{~cm}$.

\section{Stimuli and Procedure.}

We measured the temporal change in the accuracy in the probe test under two conditions: (1) whether participants reported that they were aware or unaware of the arrow-cue direction and (2) whether the arrow-cue was correctly predictive for later probe position (validity). We presented the arrow-cue at two contrasts (the trial ratio of low to high contrast equalled 4 to 1) to control the masking effect. The low-contrast cue had $81 \%$ of the luminance of the high-contrast cue (Fig. 1: Cue). The masking stimulus was a picture of random RGB dots that covered the area where the cue was presented. The target memory array was presented as eight Landolt c rings, and each ring's slit (ring direction) was positioned in eight different possible directions. The eight directions were in the 12:00, 1:30, 3:00, 4:30, 6:00, 7:30, 9:00 and 10:30 o'clock directions. The ring direction was produced in pseudo-random order from the eight directions. Each ring was located equidistantly at the eight different positions, forming an imaginary circle at $<9^{\circ}$ in diameter from the centre fixation point (Fig. 1: Target memory array). The eight rings locations were the 12:00, 1:30, 3:00, 4:30, 6:00, 7:30, 9:00 and 10:30 o'clock locations. The control cue was a circle filled with the same colour and contrast as the low-contrast 
cue. The probe cue was a white-bordered square.

The experimental paradigm (Fig. 1) adapted forward and backward masking ${ }^{37}$ based on the experimental 1 diagram of Delvenne and Holt (2012). A similar method has been used in several previous studies ${ }^{29,33}$. Our paradigm had two trial types: normal type and perceptual report type. In the former, participants performed probe tests and reported their perceptual awareness, whereas in the latter, they only reported their perceptual awareness to ensure its quality in the normal trial type.

All participants had some practice sessions and six test sessions. In the practice sessions, the participants trained to understand the behavioural paradigm. To help them understand the experimental paradigm, we used only high-contrast and valid arrow-cues in practice sessions. In test sessions, low contrast arrow-cues were used with $80 \%$ probability, and the remaining cues were high contrast, with a mixture of valid, invalid and control arrow-cues (ratio of valid:invalid:control trials $=3: 1: 1$ ). Each session included 100 trials and usually took 1.5 hours for the six test sessions for one participant.

In normal trials, each trial started with a central fixation cross that was presented for 800-1500 ms. Following the fixation cross, three pre-masks were presented for $48 \mathrm{~ms}$ (each $16 \mathrm{~ms}$ ). After the pre-mask presentation, an arrow-cue directed toward one of eight locations was presented for $16 \mathrm{~ms}$, followed by three post-masks (same as pre-mask presentation). The target memory array was then presented for $67 \mathrm{~ms}$ after a 32-952 ms delay following the flashing of the post-masks, followed by a $100 \mathrm{~ms}$ blank interval and by a probe that remained present until the response key was pressed. After the probe response, participants made an unspeeded response to report their perceptual awareness of the arrow-cue, whether the direction of the arrow-cue was seen clearly and was discriminable (fully visible), seen obscurely and was indiscriminable (fragmented visible), or not seen at all (invisible), by pressing the G, H or J key, respectively.

The perceptual awareness report trial had the same procedure before the target memory array presentation. After the delay, participants responded with their unspeeded perceptual awareness report without the probe test.

The conditions were randomly changed during the experiment. All trials started after pressing the keyboard in the perceptual awareness report of the previous trial. In one session (100 trials), a normal trial type included 90 trials, with 54 trials counted as valid, 18 trials as invalid, 18 trials as control arrow-cue presentations, and the remaining 10 trials as perceptual awareness trials. The delay was selected randomly and independently with a duration of $83,116,150,183,250,333,433,566,750$, or $1000 \mathrm{~ms}$ (equally spaced on a logarithmic scale). 


\section{Results}

First, the accuracy in the probe test irrespective of the delay was calculated (Fig. 2A). We performed Tukey's pair-wise post hoc test after two-way analysis of variance (ANOVA) with awareness of arrow-cue direction and three arrow-cue direction types (validity and control). The participants performed significantly better in valid-aware and valid-unaware conditions compared with control and invalid conditions ( $p<0.001$ and $\mathrm{p}<0.001$, respectively). In contrast, accuracy did not differ significantly between the control and two invalid conditions. The reaction time was only significantly shorter in the valid-aware condition compared to the other conditions (Fig. 2B, p $=0.004$ with validunaware, $\mathrm{p}=0.001$ with control and invalid-aware and $\mathrm{p}<0.001$ with invalid-unaware).

We therefore explored the relationships of the accuracy and reaction time with the delay, i.e., the inter-stimulus interval between the presentation of the arrow-cue and target memory array (Fig. 3A-B). The accuracy was 75-90\% in the valid-aware condition (Fig. 3A, blue line) and gradually increased upon increasing the delay in the control condition (Fig. 3A, black line). In contrast, time-dependent changes were observed in the other conditions (Fig. 3A, red, purple and green lines). The reaction time in the validaware condition was significantly shorter regardless of the delay compared to the other conditions, but the other conditions did not differ significantly based on the delay (Fig. 3B).

We next addressed the temporal dynamics of the endogenous attentional effect on behavioural performance. We defined delta as the difference in accuracy between the valid-unaware and control (the value of valid-unaware minus control). The accuracy exhibited a clear peak at approximately $333 \mathrm{~ms}$ after presentation of the arrow-cue (Fig. 4A). The reaction time also had the lowest delta for the $333 \mathrm{~ms}$ delay (Fig. 4B). We then analysed the correlation between these deltas, and there was a significant negative correlation (Fig. 4C, Pearson's $r=-0.768, p=0.010$ ).

Finally, we classified the delay time into three phases to analyse the timedependent changes in longer time windows: 83-150, 183-333 and 433-1000 ms. The classification sizes were based on the significantly better accuracy between valid-aware and control conditions in three consecutive delay times (183, 250 and $333 \mathrm{~ms}$, see Fig. $3 \mathrm{~A})$. Accuracy was significantly greater in the intermediate phase compared to the first and last phases (Fig. 4C, $p=0.024$ vs. first phase and $p<0.001$ vs. last phase). The reaction time in the intermediate phase was significantly decreased compared to the last phase (Fig. 4D, p = 0.014) and was also decreased compared to the last phase with a significant trend (Fig. 4D, $\mathrm{p}=0.069$ ). 


\section{Discussion}

Many previous studies exploring the unconscious modulation of our cognitive performance by unconscious visual information have focused on the dissociation between reports of subjective cue awareness and actual performance. The quest for neural mechanisms for the unconscious modulation implies that how the dissociation changes through the specific time windows is quite important to understand the neural representation or processing of it.

As an approach to answer this question, we examined the temporal dynamics of the endogenous attentional effect by measuring the transfer of the visual stimulus to VSTM while manipulating the awareness of arrow-cue directions. Our results reveal that endogenous attention was elicited irrespective of cue awareness, as previous studies reported (Fig. 2A), and significantly better performance was observed with 83, 183, 250 and $333 \mathrm{~ms}$ delays when participants reported being unaware of arrow-cue direction (Fig. 3A). We also analysed the dissociation distance (i.e., delta) between valid-unaware and control conditions using accuracy and reaction time as measures of the endogenous attentional effect. The delta of the accuracy formed the shape of an arch, with a peak at $333 \mathrm{~ms}$ (Fig. 4A). The delta of the reaction time showed the reverse trend, with the lowest value at $333 \mathrm{~ms}$ (Fig. 4B). There was a significant negative correlation between these deltas (Fig. 4C, Pearson's $r=-0.768, p=0.010$ ). This indicates that the endogenous attention affects cognitive performance in a time-dependent manner.

To address its time-dependent manner, we divided the delays into three phases due to the significantly better accuracy in the 183, 250, and $333 \mathrm{~ms}$ delay conditions (Fig. $3 \mathrm{~A})$. The intermediate phase showed significant differences compared to first and last phases (Fig. 4D, E). This suggests that endogenous attention elicited by an unconscious arrow-cue could affect performance during specific time windows. In contrast, endogenous attention elicited by a conscious arrow-cue maintained its effect regardless of the delay. These results suggest that endogenous attentional modulation was stronger at 183-333 ms delays and did not occurred at longer delays (433 $\mathrm{ms}$ and longer) in this study.

One model for explaining the different time-dependent results between validaware, valid-unaware and control conditions is a cross of temporal expectation and attentional scope. Temporal expectation has often been considered a hazard function of the presentation of the imminent target stimuli ${ }^{38,39}$. In our study, a gradual increase in the accuracy with increasing delay was observed in valid-aware and control conditions. This implies that participants could manipulate their attention to target memory array presentation with expectancy because the later delay caused a higher incidence of target 
presentation in their expectation. Visual attentional scope for the visual local field enhances the visual processing ${ }^{40,41}$. The scope was only covered with a Landolt $\mathrm{c}$ ring instructed by the arrow-cue in the valid-aware condition, while the scope was covered with the target memory array in the control condition. This difference in visual attentional scope could grade the accuracy in the valid-aware condition. Then, the temporal dynamics of accuracy in the valid-unaware condition could be expected to be located intermediately between the valid-aware and control conditions, with a gradual increase. However, a gradual increase in accuracy was not observed in the valid-unaware trials in our study. This suggests that attentional modulation, triggered by unconscious arrow-cue direction, has different functional attributions from the cross of temporal expectancy and visual attentional scope.

Finally, the present study supports the notion that endogenous attention is occurred by unconscious visual stimuli ${ }^{29,30}$. Functional brain imaging studies have shown that endogenous visual attention modulates visual processing ${ }^{42,43}$. Our findings and these previous studies offer the opportunity to explore the temporal process of the endogenous visual attention with consciousness of the visual cue-stimulus as an independent variable. Such experiments may shed light on the neural correlates of consciousness ${ }^{44}$.

\section{References}

1. Clifford, C. W. G. \& Harris, J. A. Contextual modulation outside of awareness. Curr. Biol. 15, 574-578 (2005).

2. He, S. \& MacLeod, D. I. Orientation-selective adaptation and tilt after-effect from invisible patterns. Nature 411, 473-476 (2001).

3. Dehaene, S. et al. Imaging unconscious semantic priming. Nature 395, 597-600 (1998).

4. $\quad$ van Gaal, S., Ridderinkhof, K. R., Fahrenfort, J. J., Scholte, H. S. \& Lamme, V. A. F. Frontal Cortex Mediates Unconsciously Triggered Inhibitory Control. $J$. Neurosci. 28, 8053-8062 (2008).

5. Campion, J., Latto, R. \& Smith, Y. M. Is blindsight an effect of scattered light, spared cortex, and near-threshold vision? Behav. Brain Sci. 6, 423-448 (1983).

6. Weiskrantz, L. Blindsight: a Case Study in its Implications. (1986).

7. Tong, F. Primary visual cortex and visual awareness. Nat Rev Neurosci 4, 219 229 (2003).

8. Macknik, S. L. \& Martinez-Conde, S. Dichoptic Visual Masking Reveals that Early Binocular Neurons Exhibit Weak Interocular Suppression: Implications for Binocular Vision and Visual Awareness. J. Cogn. Neurosci. 16, 1049-1059 
(2004).

9. Tong, F., Nakayama, K., Vaughan, J. T. \& Kanwisher, N. Binocular Rivalry and Visual Awareness in Human Extrastriate Cortex. Neuron 21, 753-759 (1998).

10. Rees, G., Russell, C. \& Driver, J. Inattentional Blindness Versus Inattentional Amnesia for Fixated But Ignored Words. Science (80-. ). 286, 2504-2507 (1999).

11. Bonneh, Y. S., Cooperman, A. \& Sagi, D. Motion-induced blindness in normal observers. Nature 411, 798-801 (2001).

12. Tsuchiya, N. \& Koch, C. Continuous flash suppression reduces negative afterimages. Nat Neurosci 8, 1096-1101 (2005).

13. Gaillard, R. et al. Nonconscious semantic processing of emotional words modulates conscious access. Proc. Natl. Acad. Sci. 103, 7524-7529 (2006).

14. Naccache, L. \& Dehaene, S. Unconscious semantic priming extends to novel unseen stimuli. Cognition 80, 223-237 (2001).

15. Dijksterhuis, A., Bos, M., F Nordgren, L. \& Baaren, R. On Making the Right Choice: The Deliberation-Without-Attention Effect. Science (New York, N.Y.) 311, (2006).

16. Bargh, J. A., Chen, M. \& Burrows, L. Automaticity of social behavior: Direct effects of trait construct and stereotype activation on action. J. Pers. Soc. Psychol. 71, 230-244 (1996).

17. Azzopardi, P. \& Cowey, A. Blindsight and visual awareness. Conscious. Cogn. 7, 292-311 (1998).

18. Pessiglione, M. et al. How the Brain Translates Money into Force: A Neuroimaging Study of Subliminal Motivation. Science (80-. ). 316, 2006-2008 (2007).

19. Posner, M. I. Orienting of attention. Q. J. Exp. Psychol. 32, 3-25 (1980).

20. Gandhi, S. P., Heeger, D. J. \& Boynton, G. M. Attentional Modulation Strength in Cortical Area MT Depends on Stimulus Contrast. Proc. Natl. Acad. Sci. U. S. A. 96, 3314-3319 (1999).

21. Liu, T., Larsson, J. \& Carrasco, M. Feature-based Attention Modulates Orientation-Selective Responses in Human Visual Cortex. Neuron 55, 313-323 (2007).

22. Liu, T., Pestilli, F. \& Carrasco, M. Transient attention enhances perceptual performance and fMRI response in human visual cortex. Neuron 45, 469-477 (2005).

23. Martinez-Trujillo, J. C. \& Treue, S. Attentional Modulation Strength in Cortical Area MT. Neuron 35, 365-370 (2002). 
24. Hsieh, P. J., Colas, J. T. \& Kanwisher, N. Pop-out without awareness: Unseen feature singletons capture attention only when top-down attention is available. Psychol. Sci. 22, 1220-1226 (2011).

25. Mccormick, P. Orienting Attention Without Awareness. Journal of experimental psychology. Human perception and performance 23, (1997).

26. Mulckhuyse, M., Talsma, D. \& Theeuwes, J. Grabbing attention without knowing: Automatic capture of attention by subliminal spatial cues. Vis. cogn. 15, 779-788 (2007).

27. Tan, J. \& Hsieh, P. Eye-specific information biases perceived direction of bistable motion. J. Vis. 13, 1-8 (2013).

28. Van der Stigchel, S., Mulckhuyse, M. \& Theeuwes, J. Eye cannot see it: The interference of subliminal distractors on saccade metrics. Vision Res. 49, 2104 2109 (2009).

29. Palmer, S. \& Mattler, U. Masked stimuli modulate endogenous shifts of spatial attention. Conscious. Cogn. 22, 486-503 (2013).

30. Palmer, S. \& Mattler, U. On the source and scope of priming effects of masked stimuli on endogenous shifts of spatial attention. Conscious. Cogn. 22, 528-544 (2013).

31. Makovski, T. \& Jiang, Y. V. Distributing versus focusing attention in visual short-term memory. Psychon. Bull. Rev. 14, 1072-1078 (2007).

32. Schmidt, B. K., Vogel, E. K., Woodman, G. F. \& Luck, S. J. Voluntary and automatic attentional control of visual working memory. Percept. Psychophys. 64, 754-763 (2002).

33. Delvenne, J. F. \& Holt, J. L. Splitting attention across the two visual fields in visual short-term memory. Cognition 122, 258-263 (2012).

34. Brainard, D. H. The Psychophysics Toolbox. Spat. Vis. 10, 433-436 (1997).

35. Boy, F., Husain, M. \& Sumner, P. Unconscious inhibition separates two forms of cognitive control. Proc. Natl. Acad. Sci. 107, 11134-11139 (2010).

36. Reuss, H., Desender, K., Kiesel, A. \& Kunde, W. Unconscious Conflicts in Unconscious Contexts: The Role of Awareness and Timing in Flexible Conflict Adaptation. Journal of experimental psychology. General 143, (2014).

37. Janczyk, M. \& Reuss, H. Only pre-cueing but no retro-cueing effects emerge with masked arrow cues. Conscious. Cogn. 42, 93-100 (2016).

38. Coull, J. T. Neural substrates of mounting temporal expectation. PLoS Biol. 7, 8 11 (2009).

39. Nobre, A., Correa, A. \& Coull, J. The hazards of time. Curr. Opin. Neurobiol. 
17, 465-470 (2007).

40. Eriksen, C. W. \& james, J. D. Visual attention witnin and around the field of focal attention: a zoom lens model. Percept. Psychophys. 40, 225-240 (1986).

41. Müller, N. G., Bartelt, O. A., Donner, T. H., Villringer, A. \& Brandt, S. A. A physiological correlate of the 'Zoom Lens' of visual attention. J. Neurosci. 23, 3561-3565 (2003).

42. Buschman, T. J. \& Miller, E. K. Top-down versus bottom-up control of attention in the prefrontal and posterior parietal cortices. Science (80-. ). 315, 1860-1862 (2007).

43. Corbetta, M. \& Shulman, G. L. Control of goal-directed and stimulus-driven attention in the brain. Nat. Rev. Neurosci. 3, 201-215 (2002).

44. Crick, F. \& Koch, C. Consciousness and Neuroscience. Cereb. Cortex 97-107 (1998).

\section{Acknowledgements}

This research was supported by Research Grant KAKENHI $17 \mathrm{H} 01769$ (to K.K.) and 15J04452 (to S.M.). We thank Edward William Ko Uy for helpful comments on the manuscript. We would like also to thank Yoshio Sakurai for helpful discussion.

\section{Author contributions}

Y.O., S.M., J.S. and K.K. designed the experiments. Y.O., S.M. and J.S. performed the experiments and analysed the data. K.K. supervised the project. All authors contributed to discussion. Y.O. and K.K. wrote the manuscript.

\section{Conflict of Interest Statement}

The authors declare that the research was conducted in the absence of any commercial or financial relationships that could be construed as a potential conflict of interest. 


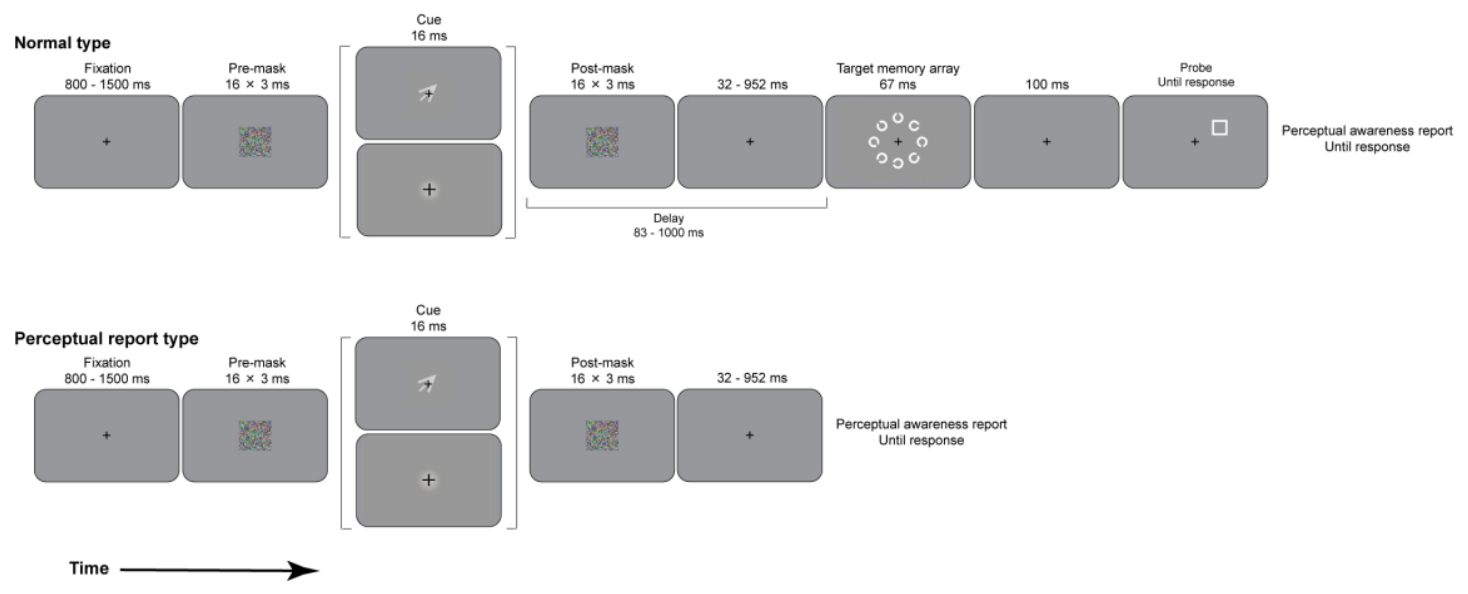

Figure 1. Schematics of sequences of the behavioural paradigm

(Upper) Schematic of a normal trial. In each trial, a fixation cross was presented for 800$1500 \mathrm{~ms}$, followed by three pre-masks. After the pre-mask presentation for $48 \mathrm{~ms}$, an arrow-cue or circle-cue was presented for $16 \mathrm{~ms}$, followed by post-mask presentation in the same way as the pre-mask presentation. Following a $32-952 \mathrm{~ms}$ delay, the target memory array was presented for $67 \mathrm{~ms}$. Participants were instructed to transfer it into their visual short-term memory and answer the probe test after the retention interval (100 $\mathrm{ms}$ ). After the probe test, they performed an unspeeded perceptual report. (Lower) Schematic of the perceptual report type. The equal sequences before the target memory array presentation in a normal trial. In this trial type, the participants performed only the perceptual report after a 32-952 ms delay. 
A

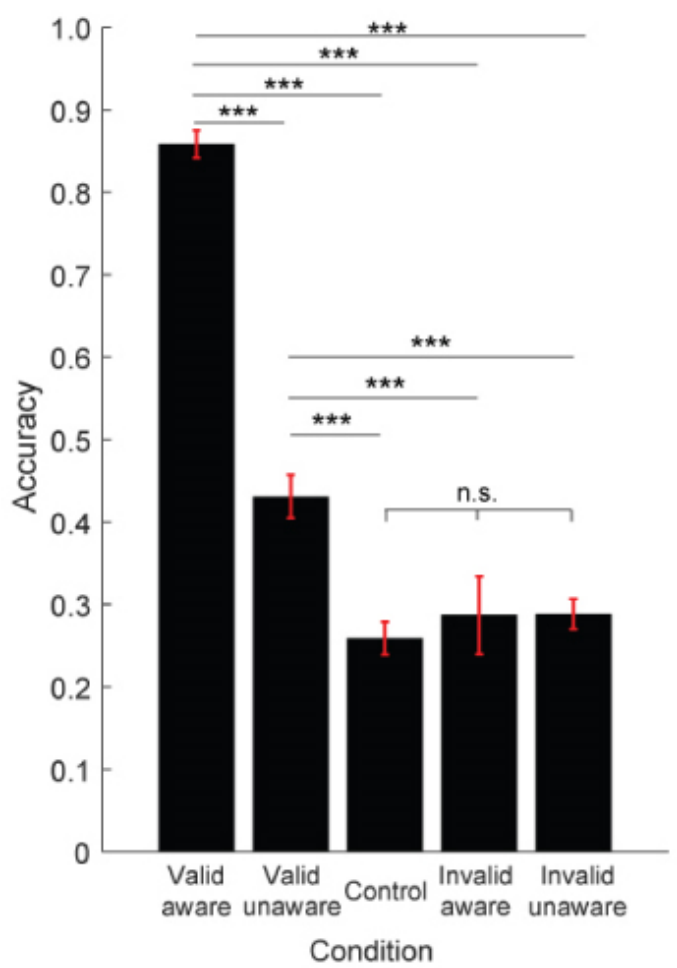

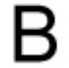

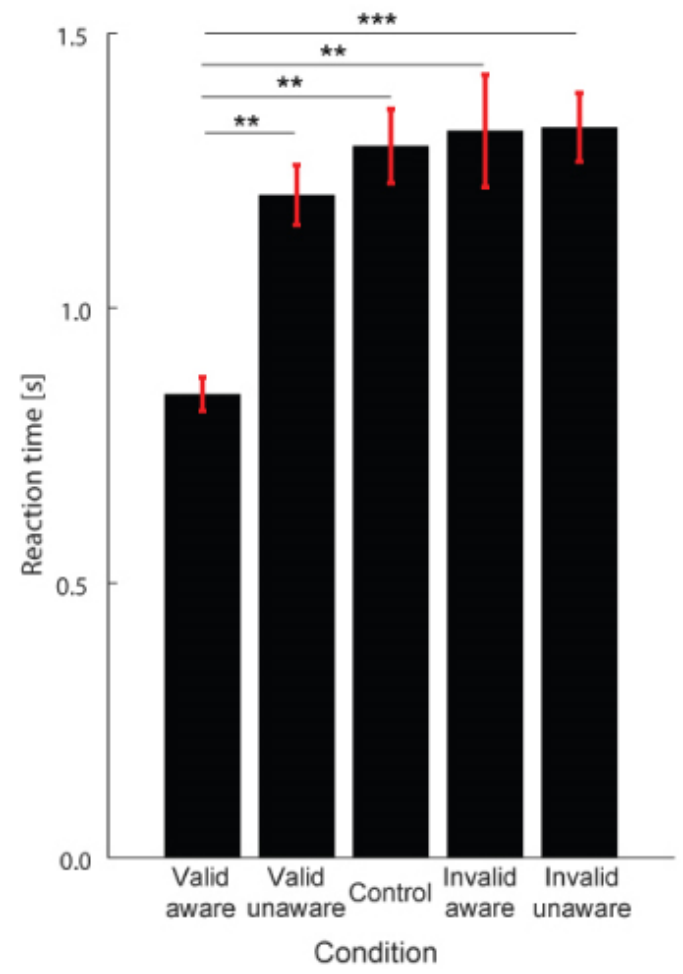

Figure 2. Behavioural performance of the pre-cue paradigm

(A) The accuracy of the probe test is shown as the mean \pm standard error of the mean (SEM). (B) The reaction time of the probe test is shown as the mean $\pm \mathrm{SEM}$. ${ }^{* *} \mathrm{p}<0.01$, $* * * \mathrm{p}<0.001$. 
A

\section{B}
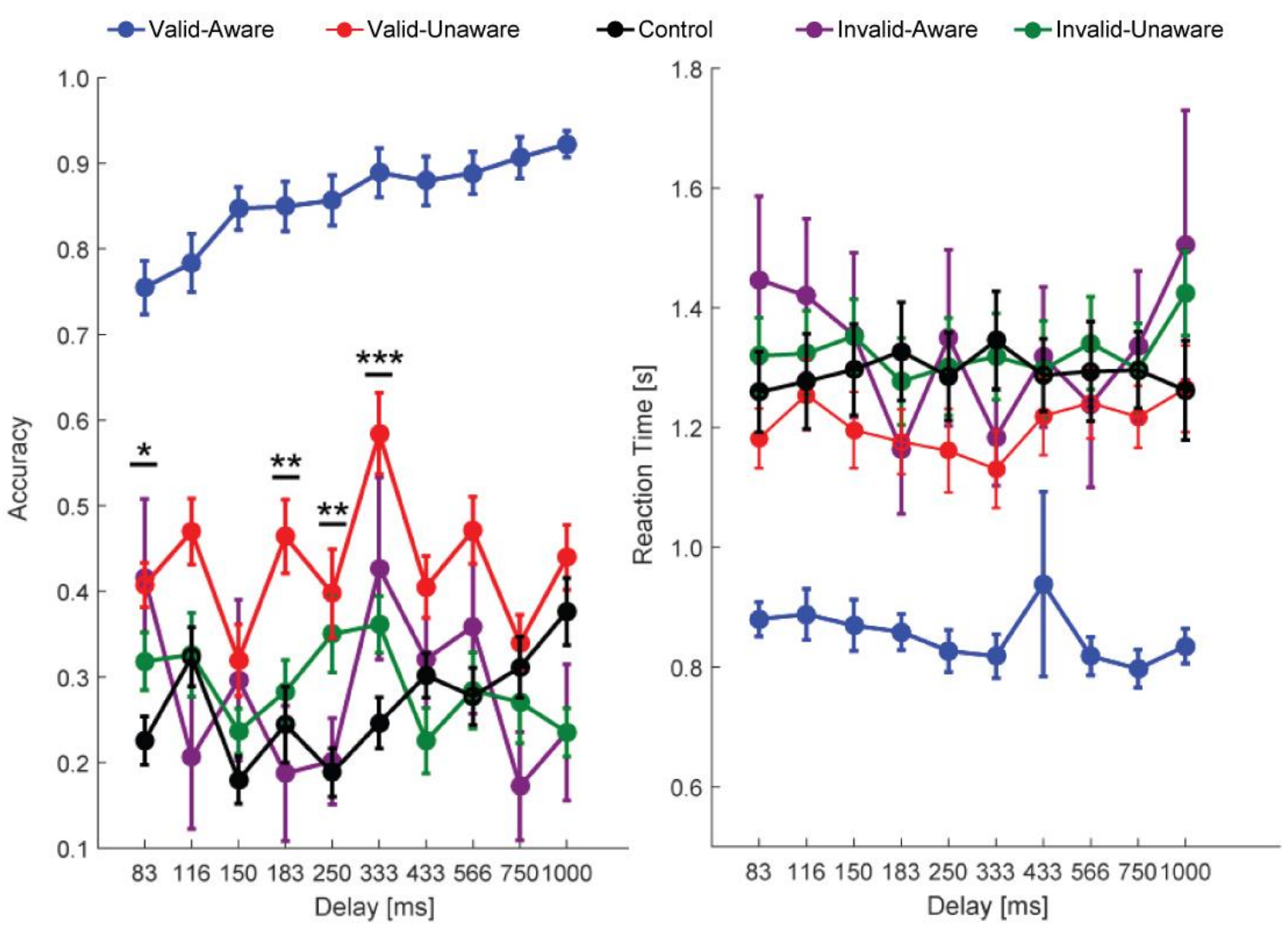

Figure 3. Temporal changes in behavioural performance.

(A) Temporal change in accuracy in the probe test. Each line shows the mean \pm SEM. (B) Temporal change in reaction time in the probe test. Each line shows the mean \pm SEM. $* \mathrm{p}<0.05, * * \mathrm{p}<0.01, * * * \mathrm{p}<0.001$. 

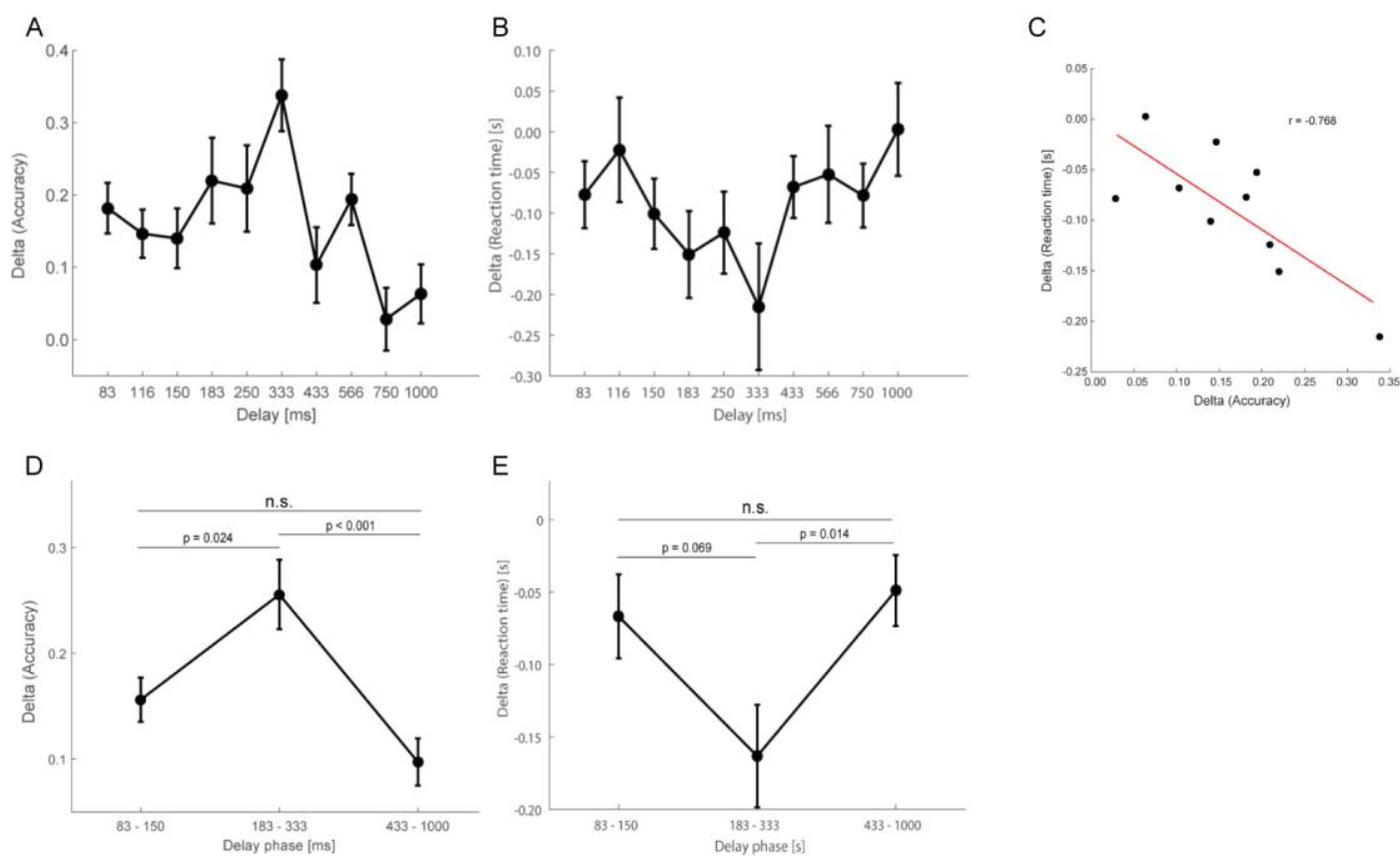

\section{Figure 4. Temporal changes in the delta}

(A) Temporal change in the delta of the accuracy is shown as the mean $\pm \mathrm{SEM}$. (B) Temporal change in the delta of the reaction time is shown as the mean \pm SEM. (C) Significant correlation between the delta of the accuracy and the delta of the reaction time (Pearson's correlation, $r=-0.768, \mathrm{p}=0.010$ ). (D) Temporal change in the delta of the accuracy in large time windows is shown as the mean \pm SEM. (E) Temporal change in the delta of the reaction time in large time windows is shown as the mean $\pm \mathrm{SEM}$. 